\title{
Conception du drainage dans les barrages en terre
}

\author{
par G. Post et P. Guerber \\ Bureau d'Etudes Coyne et Bellier, \\ Paris
}

\section{1. - Introduction}

Les accidents attribués aux infiltrations et aux érosions qu'elles entraînent ont heureusement diminué au cours des dernières décennies; ils n'en restent pas moins encore trop fréquents et particulièrement dangereux même si, grâce à une surveillance constante rendant possible une intervention rapide, ils n'ont généralement pas provoqué de ruptures totales.

Terzaghi a justement constaté, il y a déjà plus de vingtcinq ans, que "les ruptures réellement catastrophiques sont celles dues aux érosions internes par infiltration ou renards, du fait qu'elles se produisent sans avertissement, le réservoir étant plein et parfois plusieurs années après le premier remplisage».

Ces infiltrations dangereuses, qui finissent par produire une érosion interne du barrage ou des fondations, peuvent avoir des origines très diverses:

- fissuration due à des tassements différentiels comme aux barrages de Wister [1] (U.S.A.) en 1949, et de East Branch [1] (U.S.A.) en 1957;

- fuites à travers la fondation, au contact avec le noyau comme à Hills Creek [1] (U.S.A.) en 1970, ou dans une rive comme à Fontenelle [2] (U.S.A.) en 1965;

- fissuration horizontale due à des transferts de charge du noyau sur les massifs amont et aval et à un claquage ou fracturation hydraulique des zones peu comprimées comme à Hyttejuvet [3] (Norvège) en 1966 et à Balderhead [4] (Angleterre) en 1967;

- entraînements de fines à la suite d'élongation et de rupture de tuyaux enterrés collecteurs de puits filtrants, comme à Grenada[1] (U.S.A.) en 1954;
- tassements différentiels près d'une faille, d'une fondation compressible particulièrement érodable, comme à Baldwin Hills [5] (U.S.A.) en 1963.

A ces causes mécaniques s'ajoute souvent une cause physico-chimique encore assez mal connue, la défloculation de certaines argiles [6].

\section{2. - Les enseignements des accidents}

Tous ces ouvrages avaient déjà fait l'objet de reconnaissances sérieuses il y a quinze à vingt-cinq ans, et si les méthodes et appareillages disponibles actuellement avaient existé ils auraient sans doute permis un peu plus de précision dans la connaissance des fondations et le contrôle des remblais, mais on ne peut affirmer que cela aurait suffi pour éviter ces accidents. Seule une conception différente du drainage, née de ces observations et d'autres similaires, doit permettre de faire face à l'essentiel de ces comportements imprévus.

\subsection{Hétérogénéité des remblais et de la fondation.}

Un barrage en terre n'est pas constitué de zones isotropes, aussi bien en raison de l'hétérogénéité inévitable des matériaux que des variations de teneur en eau et de densité à la mise en place ainsi que le montrent par exemple les résultats des contrôles du barrage de Roseires [7] au Soudan. Il en résulte des variations de perméabilité importantes, dans le rapport de 10 à 100 , à l'intérieur d'une même zone, encore que la tendance actuelle soit de plus en plus de traiter une très grande partie des matériaux des remblais par 
criblage et mélange, comme au barrage de Tarbela, par exemple [8], ce qui permet d'obtenir des zones présentant des caractéristiques beaucoup plus homogènes. Au barrage du Mont-Cenis seule l'anisotropie due à la mise en place par couches minces fut constatée, la ségrégation de ce matériau morainique à granulométrie continue et pauvre en fines ( $20 \%$ environ) ayant été évitée par la mise au point des modes d'extraction et de criblage et par mise en dépôt provisoire avec reprise [9]. La perméabilité horizontale est 5 à 10 fois supérieure à la perméabilité verticale d'après les réseaux d'écoulement réels déduits des mesures faites à retenue pleine à l'aide de nombreuses cellules de pression interstitielle.

La meilleure solution pour pallier l'hétérogénéité d'un remblai en terre argileuse dit homogène, comme celui du barrage de Bir M'Cherga en Tunisie, de $43 \mathrm{~m}$ de hauteur, achevé en 1971, est certainement celle du filtre disposé au sein du corps du barrage et se retournant sous le talus aval (fig. 1 et 2) dérivée de la disposition adoptée pour la première fois par Terzaghi pour le barrage de Vigario en 1947 au Brésil.

Ce type de drainage interne présente également l'avantage très important d'intercepter toute fissuration du remblai et de collecter les fuites qui pourraient s'y produire.

Le même dispositif a été utilisé avec succès dans plusieurs barrages brésiliens pour les mêmes raisons. En particulier, des fissures et pertes d'eau ont été constatées lors de l'installation de piézomètres dans le barrage de Graminha, à mi-hauteur du remblai, par suite de tassements différentiels, au-dessus des sols résiduels de la rive gauche sans qu'aucun dommage ait été constaté depuis la mise en eau en 1965. Aucun voile d'injection ou mur parafouille n'a été prévu sur cet ouvrage, le filtre vertical se retourne simplement à l'aval de la tranchée parafouille non descendue au rocher et est prolongé par des puits filtrants non visitables selon un dispositif analogue à celui de Vigario [10].

La capacité d'évacuation de ces drains brésiliens (en général de 1,00 à $1,50 \mathrm{~m}$ de largeur), composés d'un seul matériau sableux mis en place par excavation successive dans le remblai de tranchées superposées, reste relativement faible.

Par contre, au barrage de Sasumua[11], barrage homogène en argile très plastique (Kenya), le filtre central vertical projeté par Terzaghi comporte trois couches, d'ail leurs extrêmement minces, mis en place à l'aide de coffrages glissant entre les couches.

Parfois le drain est incliné vers l'amont, améliorant ainsi la stabilité lors de la vidange. C'est le cas, par exemple,

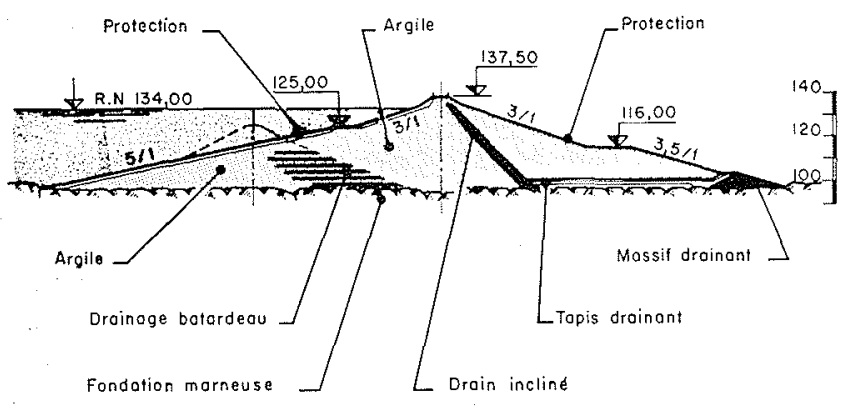

1/ Bir M'Cherga. Coupe type. du barrage de North Hartland (U.S.A.) et celui de la digue de la Morge (France).

Une attention toute particulière doit être apporiée au drainage autour des conduites sous remblai. Au barrage de Bir M'Cherga, la conduite de l'évacuateur de crues de $5,5 \mathrm{~m}$ de diamètre est entourée, à l'aval du drain incliné, par un filtre à deux couches, afin d'éliminer toute fuite susceptible de se développer au contact du béton.

\subsection{Les risques de fissuration et la fracturation hydrauli- que.}

\subsubsection{DÉFORMATIONS ET FISSURATION.}

Les causes pouvant entraîner des tractions dans le corps du remblai étanche, et créer des fissures plus ou moins ouvertes ou prêtes à s'ouvrir sous l'effet de l'eau de la retenue, sont très nombreuses [8].

Ce sont d'abord les tassements différentiels pendant et après la construction, qu'ils soient dus à la fondation irrégulière de par sa topographie ou de par sa nature, ou aux compressibilités différentes et différées des remblais des différentes zones. Les phases de construction et notamment la construction tardive d'une section du barrage réservée au passage de la rivière sont également l'origine de tassements différentiels souvent importants.

$\mathrm{Au}$ cours de la construction des remblais argileux des couches trop exposées à la dessiccation peuvent se fissurer finement, le défaut passant inaperçu sans qu'il y soit apporté remède.

Mais si des fissures peuvent s'être développées avant la mise en eau, de nombreuses autres causes de déformations peuvent contribuer à créer des zones faiblement comprimées ou même tendues sans qu'il y ait fissuration.

Ce sont notamment les déformations dues au remplissage et à la vidange du réservoir, celles dues à la saturation de certains matériaux (enrochements insuffisamment arrosés ou compactés, silts argileux compactés trop secs), aux transferts de charge entre un noyau argileux relativement mince et des recharges latérales peu compressibles ou autour d'un saillant rocheux ou d'un point dur dans la fondation, comme en constitue par exemple une conduite passant sous les remblais.

Des barrages à noyau central vertical ou légèrement incliné vers l'amont ont été construits à de très nombreux exemplaires ces dernières décennies; les noyaux étant souvent relativement très minces, ces barrages sont particulièrement sensibles aux transferts de charge à moins que soit organisée une variation continue de la compressibilité des différentes zones, réalisable, par exemple, en préparant le noyau avec le même matériau que les recharges encadrantes, enrichi simplement en fines pour obtenir l'étanchéité voulue [8].

Des effets d'arc peuvent se produire entre les rives abruptes d'une vallée étroite, même avec des noyaux épais.

Enfin les secousses sismiques, en entraînant des déformations et tassements différentiels, peuvent créer sinon des fissures ce qui apparaît toutefois comme un risque non négligeable, du moins des zones "tendues".

Il est suffisant de connaître approximativement (à l'aide de calculs par éléments finis par exemple) les zones d'extension ou de tassements différentiels pour renforcer les filtres et drains dans ces régions. Il faut bien dire que le jugement de l'homme de l'art et la référence aux mesures de déformations effectuées sur de nombreux barrages 
complètent de façon indispensable les résultats du calcul et souvent même les remplacent.

\subsubsection{LE « CLAQUAGE 》 OU LA FRACTURATION HYDRAULIQUE.}

Ce phénomène bien connu des injecteurs, qui l'utilisent parfois pour traiter certains terrains finement fissurés, se produit aussi dans les sols. Il a été mis en évidence involontairement lors de l'exécution de forages pour l'installation de piézomètres dans le noyau de plusieurs barrages, notamment au cours des travaux de reconnaissance de la fissuration du noyau du barrage de Djatiluhur (Indonésie) [12].

La fissure potentielle peut ne pas exister avant la mise en eau, mais si la contrainte totale principale minimum est inférieure à la pression due au remplissage du réservoir (zone peu comprimée ou même tendue ou desséchée) une fissure peut s'ouvrir et se propager jusqu'à traverser un noyau mince. En fait, ce phénomène extrêmement complexe, car il implique une redistribution des contraintes [13], demande à être étudié plus avant, mais il est fort probable qu'il a joué un rôle dans l'apparition des fuites constatées lors du premier remplissage des barrages de Hyttejuvet [3], Balderhead [4] et dans les ruptures de nombreux petits barrages homogènes [14].

\subsection{Les érosions et "renards" à travers les remblais en argile dispersive.}

De nombreux petits barrages homogènes pourtant construits suivant les règles de l'art se sont rompus par renard ou plutôt par érosion du remblai constitué d'argile ayant des propriétés dispersives. Cela a notamment été mis en évidence en Australie par Aitchison (1965) et en Israël par Kassif (1967).

On voit en effet (fig. 3) une corrélation certaine entre le pourcentage de cation sodium échangeable et les ruptures. et ceci d'autant plus que les eaux de la retenue sont plus pures. Depuis, Sherard a mis en évidence une autre corrélation plus simple, du moins valable pour les petits barrages construits avec certains sols (Oklahoma, Mississippi, Californie, Vénézuéla). Le pourcentage de cation sodium (mesuré dans un filtrat du sol étudié) par rapport aux cations totaux (sodium + calcium + potassium + magnésium) permet de délimiter les sols dispersifs a priori dangereux (fig. 4). Il y a cependant des exceptions, comme les noyaux des barrages de Hills Creek, Balderhead, Hytteiuvet. D'où la recherche d'un second critère, le pourcentage de dispersion S.C.S. (Soil Conservation Service) exprimant le rapport des pourcentages de particules inférieures à 5 microns obtenu par un essai normalisé à l'eau distillée et par l'essai standard à l'hydromètre avec défloculant. Il y aurait un risque de défloculation quand ce rapport ou pourcentage de dispersion dépasserait 40 à $50 \%$.

Il faut toutefois noter que ces corrélations sont encore floues, que les analyses hydrométriques ou sédimentométriques donnent des résultats très variables suivant le type et la quantité de défloculant et suivant la méthode (à l'hydromètre ou à la pipette) utilisés. $\mathrm{P}_{\mathrm{ar}}$ conséquent, des recherches théoriques sur la physico-chimie des argiles, aussi bien que des études statistiques plus complètes, seront nécessaires avant que l'on puisse mettre au point un essai permettant de bien déceler ce danger.

Ces recherches sont très importantes car elles peuvent avoir une influence déterminante sur la mise au point de

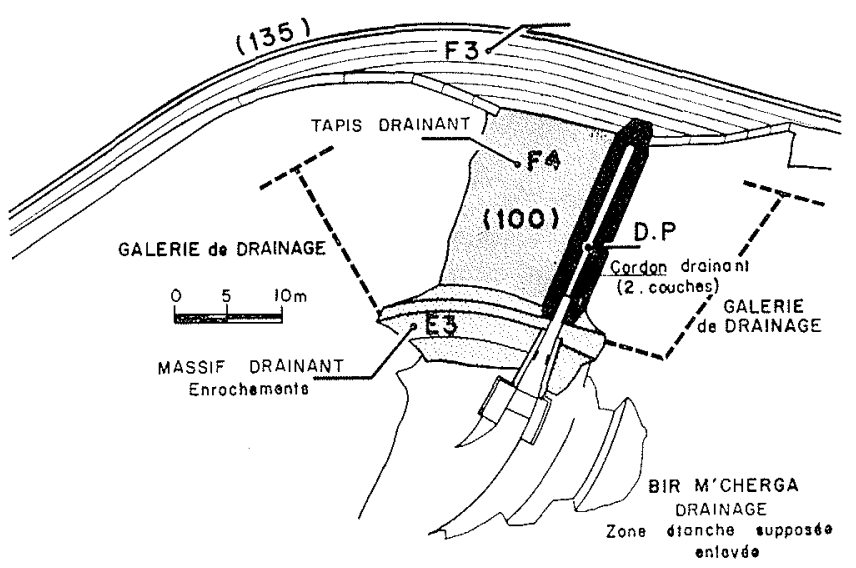

2/ Bir M'Cherga. Drainage. Zone étanche supposée enlevée.

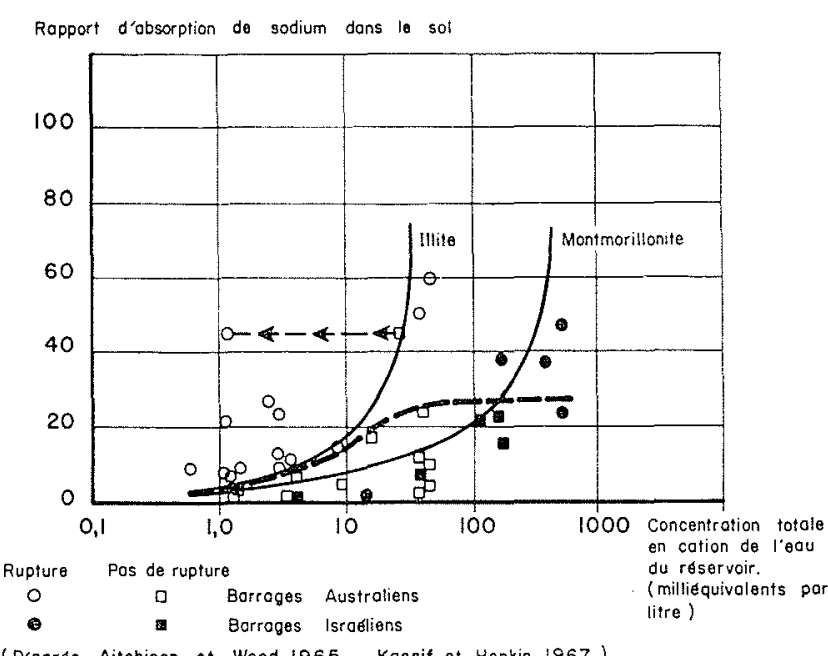

3/ Influence de la chimie des sols et des eaux sur les ruptures de petits barrages par défloculation et renard.

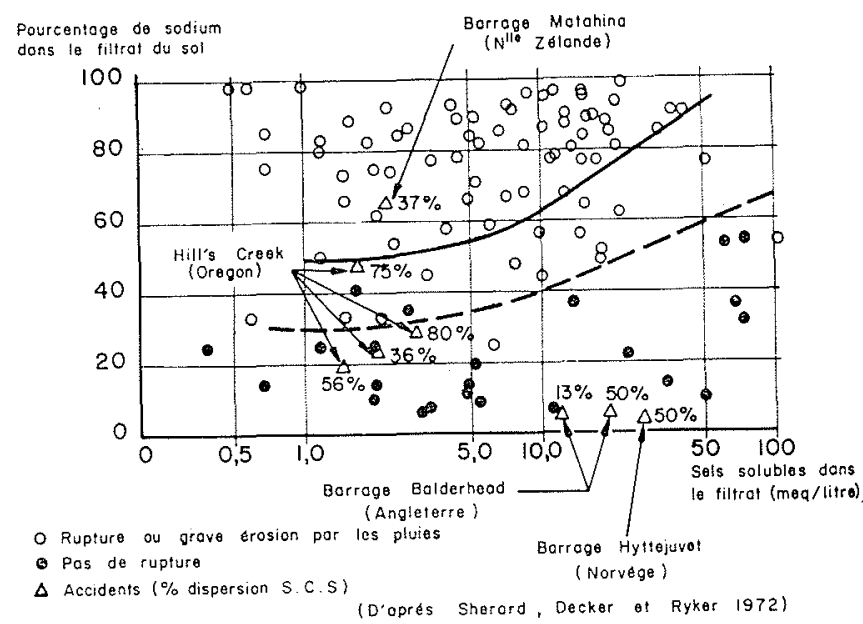

4/ Influence de la chimie des sols sur les accidents de barrages. 
nouveaux critères d'établissement de filtres permettant d'arrêter les particules d'une argile partiellement défloculée et d'éviter l'érosion interne d'un barrage [13] [14]. Ces filtres devront cependant rester non-cohérents de manière à ne pas présenter de risque de fissuration, et ne pourront donc pratiquement pas être plus fins qu'un sable de dune. Si un tel filtre ne pouvait jouer son rôle vis-à-vis d'une argile parfaitement défloculée aux grains relativement uniformes, il faudrait alors ou abandonner l'utilisation de cette argile ou la traiter chimiquement, du moins dans une zone limitée à l'amont du noyau (chaux hydratée) à condition qu'un tel traitement reste économiquement viable.

\subsection{Le drainage des fondations rocheuses.}

Il est maintenant classique, du moins selon la technique européenne, de drainer les rives des grands barrages en terre à partir de galeries recoupant les principaux accidents.

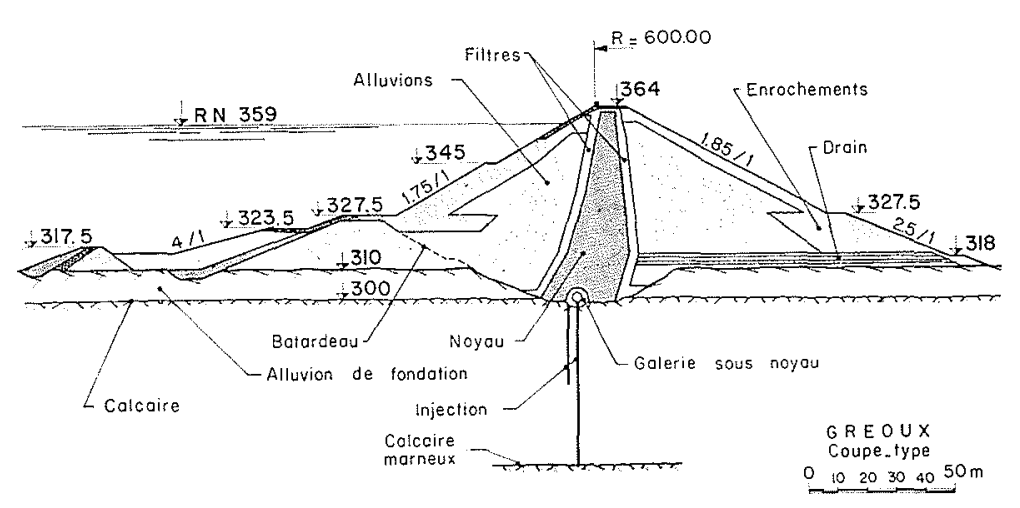

5/ Gréoux. Coupe type.

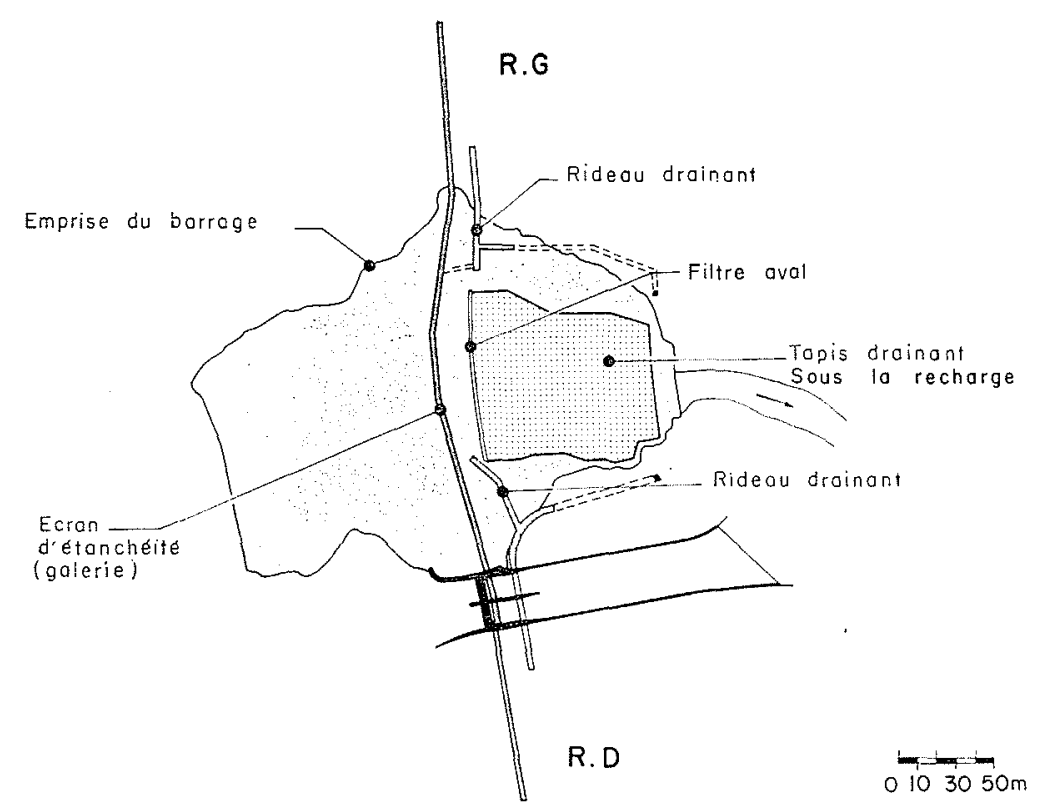

6/ Gréoux. Drainage.
Un remarquable exemple de ce type de drainage a été réalisé au barrage du Ghrib, en Algérie, voici près de trentecinq ans. De telles galeries constituent, en outre, une excellente reconnaissance complémentaire quand elles sont exécutées dès la phase du projet.

Un dispositif complet de drainage a été réalisé à Gréoux (France), barrage à noyau mince fondé au rocher calcaire. Le calcaire étant karstique, du moins au-dessus de la rivière et les recharges en alluvions et enrochements reposant sur les alluvions en place (fig. 5), on pouvait craindre qu'un karst débouche sous la recharge aval. L'examen de ce cas au moyen de réseaux d'écoulement (avec des hypothèses encadrantes) a montré la nécessité d'ajouter un excellent drain multicouche de grande capacité aux galeries de drainage dans les rives prévues pour intercepter, grâce à un rideau de forages drainants, les eaux qui pourraient mettre en charge la recharge alluviale aval (fig. 6).

Le principe est, en effet, de toujours prendre en compte la possibilité soit d'une déficience dans le voile d'injection, soit d'un accroissement de sa perméabilité avec le temps, soit d'une rupture de la paroi étanche (tranchées à la boue, paroi moulée) sous l'effet des tassements différentiels ou après une secousse sismique; le drainage assure alors à lui seul la sécurité [15] [16].

Pour cela il faut bien sûr que les organes de drainage soient visitables et que l'on puisse ausculter le fonctionnement du complexe étanchéitédrainage à l'aide d'un réseau de piézomètres disposé à l'amont comme à l'aval du dispositif d'étanchéité.

\subsection{Le drainage des barrages à masque amont.}

De plus en plus les moyens modernes de compactage (rouleaux vibrants lourds) permettent d'utiliser des enrochements de qualité médiocre (roche fissurée ou partiellement altérée) formant des remblais à granulométrie pleine, très peu compressibles, ce qui est éminemment favorable et diminue les déformations du masque amont (béton armé ou béton bitumineux). Là également l'approche du projeteur consiste à imaginer les fissures ou défauts du masque et à disposer sous ce dernier et sous le massif d'enrochements tout venant, une couche drainante de forte capacité, protégée contre la contamination des fines de l'enrochement entraînées par l'arrosage et les pluies.

Dans certains cas, comme au barrage du Vallon Dol, la prudence a été encore plus affirmée, étant donné la situation de ce barrage au-dessus de Marseille. Le massif d'enrochements est donc véritablement zoné (fig. 7), la zone amont criblée au-dessous de $100 \mathrm{~mm}$ constituant un noyau de secours par rapport aux zones aval de plus en plus grossières. Ainsi pratiquement le masque pourrait être entièrement détruit sans que le barrage en souffre, le massif étant organisé pour évacuer plusieurs $\mathrm{m}^{3} / \mathrm{s}$ sans érosion appréciable. Une expérience intéressante, quoique inattendue, a d'ailleurs prouvé au barrage du Nebaana (Tunisie) l'efficacité de cette organisation du massif en un filtre grossier. En effet, en octobre 1964, 
une crue a submergé le chantier alors que le massif aval était en avance sur le noyau incliné amont, mettant en charge de $10 \mathrm{~m}$ la face amont de ce massif, avec une dénivelée de $27 \mathrm{~m}$ jusqu'au pied aval. Très probablement grâce aux zones du massif, petits enrochements compactés à l'amont, gros enrochements déversés à l'aval, le barrage a tenu contrairement à ce qui s'est passé en 1964 pour le barrage de Hell Hole en Californie où le massif aval, entièrement en enrochements déversés fut emporté en produisant une crue artificielle de $7000 \mathrm{~m}^{3} / \mathrm{s}$.

Ces dispositions sont donc à retenir quand il y a un risque de submersion du chantier durant la construction, ce qui est bien souvent le cas.

\subsection{Le drainage au contact des ouvrages en béton, conduites ou murs de soutènement.}

Le raccordement avec ces ouvrages rigides crée toujours des points délicats pouvant favoriser la formation de fissures d'autant plus que le compactage des matériaux argileux le long des parois en béton est parfois moins efficace que dans le corps du noyau. Les matériaux les plus plastiques sont compactés nettement au-dessus de l'Optimum Proctor Standard au contact de la surface de béton préalablement parfaitement étanchée et débarrassée de tout défaut susceptible d'amorcer des cheminements privilégiés et un éventuel claquage hydraulique du noyau [17]. Pour les conduites, le drainage à l'aval du noyau d'un barrage à zones (ou à l'aval du drain incliné ou vertical d'un barrage homogène) sera constitué de deux couches de manière à obtenir une capacité d'écoulement suffisante.

Pour le contact avec le bajoyer d'un évacuateur de crues, le drainage du type adopté au barrage de Gréoux (France) ou au barrage Youssef Ben Tachfine au Maroc (fig. 8) est conçu de manière à colmater toute fissure en disposant un filtre fin spécial dépourvu de cohésion à l'amont du noyau; des filtres et drains à l'aval, avec un cordon drainant le long du bajoyer, permettent d'évacuer directement de forts débits.

D'autres solutions sont possibles lorsque le barrage en terre est raccordé à un barrage poids en béton. Dans ce cas le noyau, toujours épaissi au contact, peut envelopper complètement le béton ou bien, comme au barrage de Roseires [7], s'appuyer seulement sur la face amont et l'extrémité du barrage béton qui doit alors présenter un fruit suffisant. Les mêmes dispositions que ci-dessus relatives aux filtres amont et au drainage aval sont essentielles quant à la sécurité.

\subsection{Le projet des filtres et des drains.}

\subsubsection{LES RÈGLES GRANULOMÉTRIQUES.}

On a vu l'importance fondamentale des filtres et drains pour la sécurité. Zones relativement étroites, leur granulométrie doit être particulièrement contrôlée et sauf cas exceptionnel, les filtres et drains devront presque toujours être obtenus par criblage et lavage vigoureux.

Les fonctions granulométriques des filtres et drains, mises en lumière par Terzaghi dès 1929, aboutirent à des règles précises dès 1940 (Bertram), perfectionnées depuis par de nombreux chercheurs. Elles ne sont valables que dans certaines limites, notamment en ce qui concerne l'étendue et l'uniformité de la courbe granulométrique, et si l'on s'écarte de ces limites des essais seront nécessaires

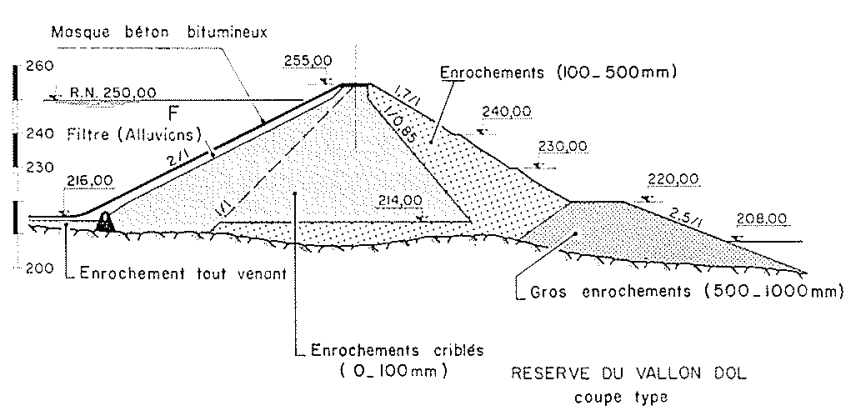

7/ Réserve du Vallon Dol. Coupe type.

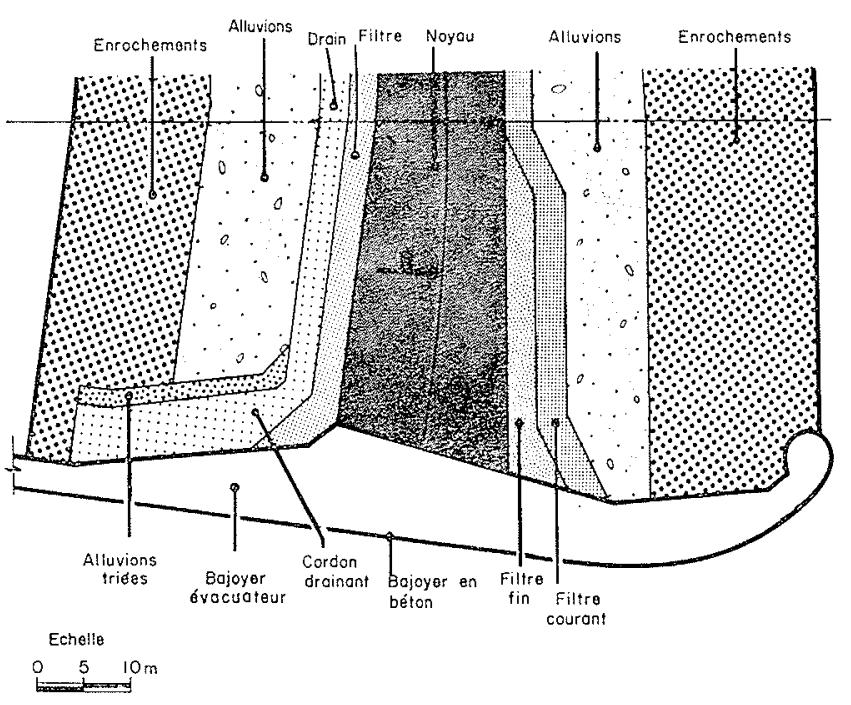

8/ Contact noyau-bajoyer. Coupe horizontale.

pour vérifier la stabilité des filtres. Les critères dépendent aussi des gradients maxima et du sens des écoulements (ascendant, descendant ou horizontal). De plus, si le filtre est soumis à des vibrations (secousses sismiques, circulation routière), on peut être amené à observer des règles plus strictes.

On ne peut cependant appliquer ces règles liant la courbe granulométrique du matériau de base à celle du filtre lorsque le matérieau de base est une argile car le filtre serait si fin qu'il deviendrait cohérent et fissurable, ce que l'on cherche absolument à éviter lorsqu'il s'agit de protéger un noyau de barrage en terre.

On a donc longtemps considéré qu'un bon sable à béton ou qu'une alluvion continue pas trop grossière assez riche en sable et propre comme celle utilisée au barrage de SerrePonçon (France) permettait de protéger convenablement à peu près toutes les argiles. Certains travaux assez récents [19] conduisirent même à àdmettre des filtres uniformes plus grossiers pour les argiles plastiques pour peu que l'interface argileuse, qui gonfle, reste légèrement comprimée. Bien entendu, tout cela n'est applicable que si le matériau argileux n'est pas dispersif [6]. Dans ce dernier cas, les critères d'étude du filtre restent à définir, mais il 
semble cependant que des sables fins devraient convenir dans beaucoup de cas lorsque l'argile défloculée comporte une partie prépondérante de particules entre 5 et $100 \mathrm{mi}$ crons.

Dans l'état actuel de nos connaissances, s'il y a un risque réel de défloculation de l'argile, un essai de filtre spécial s'impose pour en établir la granulométrie.

\subsubsection{LA PÉRENNITÉ DES FILTRES.}

Le comportement à long terme des filtres des barrages est mal connu faute d'observations suffisantes.

Il existe des risques de contamination du filtre, donc de diminution de son épaisseur et de sa capacité drainante, par migration sur une certaine longueur des fines du matériau argileux (en dehors de toute défloculation). En fait, il faut prendre une marge de quelques décimètres dans le calcul de l'épaisseur des filtres et des drains qui, d'ailleurs, sera souvent imposée par des considérations pratiques d'utilisation des engins de terrassement modernes. On pourrait craindre aussi dans les barrages de grande hauteur l'écrasement sous fortes contraintes et l'enrichissement en fines des matériaux des filtres et drains. Il semble cependant que cela doive se produire également pour les matériaux adjacents [20], à l'exception de l'argile, et que le risque ne soit pas bien grand, les règles traditionnelles entre filtres et drains restant vérifiées après cet écrasement sous contrainte.

Un autre risque, également mal connu et qui demanderait 'à être apprécié par des études de laboratoire, est celui du colmatage ou même de la cimentation des filtres par dépôt de sels métalliques. L'inefficacité de la tranchée drainante du barrage de Vermilion a été attribuée aux dépôts d'oxydes de fer. Beaucoup de noyaux sont constitués d'argile de décalcification et on pourrait craindre également un certain colmatage par dépôt de carbonate, du moins dans les zones au-dessus de la ligne de saturation.

\subsubsection{LES DIMENSIONS ET LA PERMÉABILITÉ.}

Il a été montré que l'épaisseur des filtres et drains ne pouvait être simplement déterminée en prenant en compte seulement les données des écoulements abordables par le calcul et qu'il était essentiel d'évaluer, à partir de précédents, les risques de fissures susceptibles d'entraîner un accroissement considérable des débits, ce qui conduit très souvent, du moins pour les grands ouvrages, à recourir à des filtres multi-couche.

En ce qui concerne la perméabilité, il faut noter qu'elle diminue rapidement avec le pourcentage de fines. En limitant, par lavage, ce pourcentage on peut multiplier par 10 ou même 100 la perméabilité de matériaux à granulométrie continue. Par ailleurs, il faut que le filtre aval ne soit pas traversé par la fissure lorsqu'il est saturé, ce qui exige qu'il soit dépourvu de cohésion, d'où également un très faible pourcentage de fines.

Les conséquences de la fissuration sont également annihilées par un filtre amont épais et sans cohésion. Grâce à ce filtre, la fissure ne se prolongera pas jusqu'au réservoir (une fissure ne peut rester ouverte dans un matériau pulvérulent saturé, surtout près des parements, où la contrainte moyenne est faible) et le débit se trouvera limité. Au surplus les matériaux du filtre seront entraînés par le courant et colmateront la fissure ouverte dans le matériau étanche.

Un tel filtre doit contenir un fort pourcentage de sable fin qui remplira la fissure mais sera retenu par le filtre aval. C'est pourquoi dans les zones critiques du barrage on dispose parfois un filtre spécial de sable fin interposé entre le filtre courant à granulométrie étendue et le noyau (fig. 8 et 9).

\subsubsection{LA MISE EN PLACE.}

Les conditions de mise en place ont une grande importance dans le choix des épaisseurs de filtre. Un filtre horizontal peut être plus mince qu'un filtre incliné, car il est plus facile à régler. Sauf si l'on emploie des machines spéciales pour répandre et compacter les matériaux du filtre sur la pente (exemple de Briones, U.S.A.), il faut donner à un filtre incliné ou vertical une épaisseur horizontale suffisante pour que les engins puissent rouler $(2,50$ à $4 \mathrm{~m})$. Dans le cas des filtres verticaux minces, notamment multicouches, on est amené à utiliser des coffrages de séparation, glissant à la limite des couches (exemple de Sasumua, Grand Assouan). On peut aussi creuser des tranchées superposées dans le remblai et mettre en place le filtre par couches successives de 1 à $3 \mathrm{~m}$. de profondeur.

Il est très important de limiter la contamination des couches drainantes lors de la mise en place. Des précautions particulières doivent être prises lors du répandage pour éviter la ségrégation qui peut conduire à des incompatibilités granulométriques locales. Cela est particulièrement important pour les filtres de granulométrie étendue et le long des rives ou au contact de surfaces en béton où l'on épaissit généralement les filtres. On limite généralement à 50 ou $70 \mathrm{~mm}$ la dimension maxima des éléments des filtres à granulométrie étendue et on sature le matériau pour le mettre en place de manière à réduire la ségrégation.
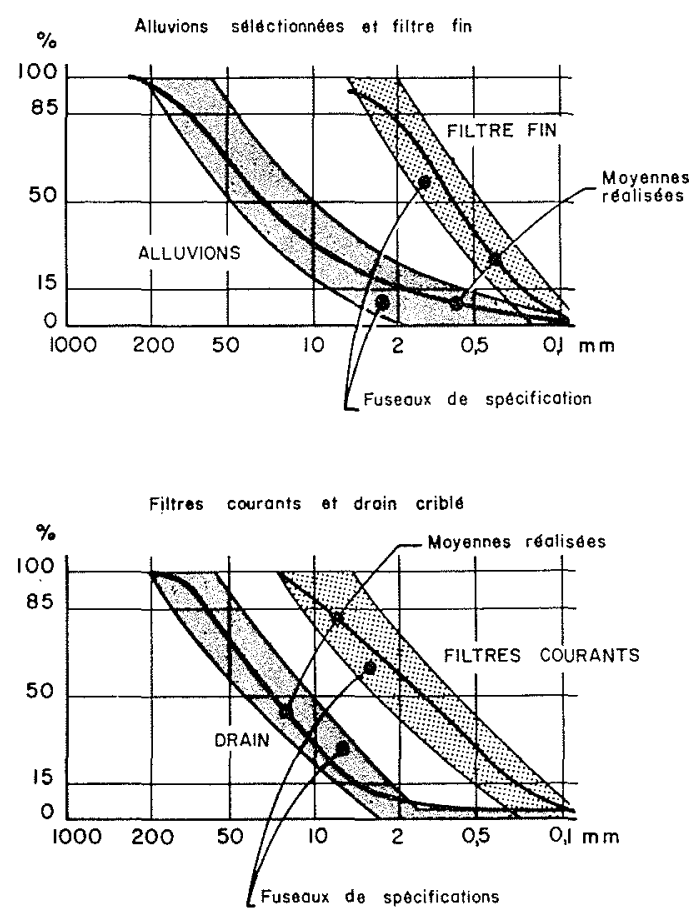

9/ Youssef Ben Tachfine. Granulométrie. 


\section{Conclusions}

La configuration des dépôts glaciaires morainiques, fluvio-lacustres ou même alluviaux est si complexe qu'il est impossible de connaître parfaitement les limites de granulométrie et de perméabilité des matériaux naturels qui seront utilisés pour la construction d'un barrage. Ces matériaux sont souvent hétérogènes et certaines de leurs propriétés, comme la défloculation des argiles dans certaines circonstances, sont mal connues. Il est donc capital de concevoir le projet de manière à pouvoir l'adapter à temps au fur et à mesure de la construction, à la meilleure connaissance que l'on acquiert des matériaux et de la fondation.

Les déformations des barrages, dues à des causes multiples (charges du remblai, dessiccation de certaines couches, irrégularités et fortes pentes des rives rocheuses, tassements différentiels et différés des remblais et des fondations, remplissage du réservoir et saturation des remblais, vidange, secousses sismiques) peuvent créer des zones d'extension ou même de fissuration des organes ou masques d'étanchéité, ce qui bouleverse les conditions initiales d'écoulement et dans certains cas, met en cause la sécurité.

On peut les réduire par un choix des matériaux des zones de transition (caractéristiques de compressibilité et de perméabilité) et par une étude détaillée des modes de mise en place et de compactage des différents matériaux.

Ces déformations ne peuvent actuellement être évaluées de façon précise mais les méthodes de calcul par éléments finis peuvent du moins situer les zones où des fissures dues aux tassements différentiels ou aux transferts de charge pourraient se produire. Il reste encore d'énormes progrès à faire pour que les puissants moyens de calcul disponibles permettent de déterminer avec précision ces zones et de calculer les dimensions de fissures, compte tenu du nombre et de la complexité des paramètres qui lient les efforts et les déformations in situ des différentes zones d'un barrage, paramètres souvent fort éloignés de ceux déterminés au laboratoire. Pour le moment, on ne peut que faire des hypothèses encadrantes et imaginer de façon tout empirique des dispositions palliant les hétérogénéités inévitables d'un barrage en terre, y compris les fissures, sans oublier les interactions, notamment sur le plan des écoulements, entre le barrage et sa fondation.

Un drainage interne doit donc être adapté au type du barrage et à sa fondation:

\section{Dans le barrage :}

- Des filtres relativement fins, rigoureusement propres, dépourvus de cohésion, seront disposés à l'amont du noyau de manière à limiter les fuites à travers les fissures, d'une part, et à colmater éventuellement ces fissures, d'autre part.

- Des filtres et drains aval devront avoir une grande capacité d'évacuation, estimée d'après les débits qui pourraient passer à travers ces fissures (souvent de l'ordre de la centaine de litres/seconde). Cela conduira souvent à un drain multi-couches dont les épaisseurs devront être suffisantes pour éviter la contamination et la ségrégation et permettre une mise en place facile avec les engins disponibles. L'exu- toire de ces drains verticaux ou inclinés se fera généralement par un tapis drainant horizontal de grande capacité souvent également multi-couches tant pour éviter la contamination par les fines venant du remblai ou des enrochements sus-jacents, que pour jouer le rôle de filtre inversé vis-à-vis de la fondation si elle est érodable.

On évitera, au moins dans les grands barrages, les tuyaux perforés collecteurs du drainage qui présentent des risques de rupture et d'élongation et peuvent entraîner le délavage et l'érosion des filtres qui les entourent.

Au droit des appuis où les dangers de fissuration ou de contournement du noyau sont particulièrement marqués, les filtres seront généralement élargis pour tenir compte également des risques de ségrégation particulièrement sérieux contre une paroi.

De même on n'oubliera pas que les conduites en béton armé qui traversent un barrage peuvent entraîner des risques tout particuliers d'érosion interne (défaut d'étanchéité des joints, fissuration du fait des tassements différentiels, la conduite constituant un point dur, défaut de compactage au contact du béton, ségrégation des matériaux) et qu'un drainage spécifique autour de ces conduites, dans leur partie aval, est absolument nécessaire.

Des précautions spéciales doivent aussi être prises le long des ouvrages en béton ou murs de soutènement limitant un déversoir et sur lesquels s'appuient les barrages en terre. La forme de la surface de contact doit être étudiée pour obtenir un bon serrage de la terre sur le béton. Ce joint particulièrement vulnérable sera muni de filtres fins épais à l'amont du noyau étanche et de filtres et drains de très grande capacité à l'aval.

Enfin, dans les régions sismiques où les risques de fissuration sont aggravés, tous les dispositifs de drainage doivent être renforcés, particulièrement dans la partie supérieure du barrage.

Pour les barrages homogènes de quelque importance, le drain vertical ou incliné à une ou plusieurs couches relié à un tapis drainant à une ou plusieurs couches, s'impose également. Les talus seront protégés contre la fissuration de retrait par des couches de sable (elles-mêmes protégées par des graviers ou enrochements) suffisamment épaisses, parfaitement propres et de grande épaisseur à l'amont, de manière à pouvoir colmater les fissures éventuelles dues aux tassements différentiels.

\section{Pour les fondations:}

On dispose de l'arsenal des tapis drainants, tranchées drainantes ou puits filtrants, sans oublier que les galeries drainantes, notamment dans les rives rocheuses, assurent une grande sécurité pour une dépense supplémentaire relativement faible vis-à-vis du coût des organes d'étanchéité dont elles permettent en même temps un bon contrôle.

Les règles des filtres habituelles sont le plus souvent bien adaptées. Pour les argiles, on évitera cependant les filtres trop grossiers, en dépit des résultats de laboratoire obtenus sur certaines argiles plastiques, jusqu'à ce qu'une meilleure connaissance des propriétés dispersives des différentes argiles permette de mieux évaluer les risques de défloculation. Pour ces argiles dispersives (pour lesquelles des règles permettant de déterminer des filtres à la fois non cohérents et efficaces restent à établir) un filtre de sable fin devrait suffire dans de nombreux cas. Des recherches 
théoriques sur les propriétés physico-chimiques des argiles, et les mécanismes d'érosion par défloculation dans une fissure, ainsi que des études statistiques portant sur les barrages où on trouve des matériaux argileux fissurés, érodables ou non, restent à faire pour préciser les essais encore incertains dont on dispose actuellement pour déceler le risque de défloculation.

Dans certains cas limites, la stabilisation des argiles défloculantes par des additifs, chaux par exemple, pourrait être une solution, de même que la modification des caractéristiques chimiques des eaux d'infiltration par l'interposition d'une couche lentement soluble (gypse par exemple) à l'amont du remblai argileux.

Des recherches sur la pérénité des filtres, leur contamination par les sels minéraux ou les carbonates, leur cimentation, leur évolution granulométrique, sont encore à faire. La publication des observations faites sur des barrages bien équipés en instruments de mesures depuis dix à vingt ans devrait aider à évaluer le comportement à long terme de ces drainages internes non visitables.

Enfin, il faut noter que, de plus en plus, les matériaux des différentes zones des barrages sont traités (criblés, lavés, mélangés), l'objectif étant de réaliser non seulement des zones de transition sur le plan de la perméabilité, mais aussi sur le plan de la déformabilité de façon à réduire les risques de fissurations horizontales ou verticales.

Un drainage interne bien implanté et largement calculé est l'organe de sécurité fondamental du barrage en terre et de sa fondation, mais un bon projet ne suffit pas à assurer la sécurité et une mise en place correcte et bien contrôlée est capitale. Le projet n'est achevé qu'après la première mise en eau et après avoir, auparavant, été adapté au fur et à mesure de la construction aux découvertes que des reconnaissances, même importantes, n'auraient pas pu révéler.

Enfin, le contrôle du comportement du drainage non seulement durant les premières mises en eau, mais tout au long de la vie du barrage, par des mesures régulières

\begin{tabular}{|c|c|c|}
\hline BARRAGE & MAÎTRE D'đTUVRE & INGÉNIEUR-CONSEIL \\
\hline $\begin{array}{l}\text { Gréoux. } \\
\text { Mont-Cenis. }\end{array}$ & Electricité de France. & Coyne et Bellier. \\
\hline Vallon Dol. & $S^{t e ́ ~}$ du Canal de Provence. & Coyne et Bellier \\
\hline Nebaana. & $\begin{array}{l}\text { Direction de l'Hydrauli- } \\
\text { que et des Aménage- } \\
\text { ments ruraux de Tuni- } \\
\text { sie. }\end{array}$ & Coyne et Bellier \\
\hline Bir M'Cherga. & $\begin{array}{l}\text { Direction de l'Hydrauli- } \\
\text { que et des Aménage- } \\
\text { ments ruraux de Tuni- } \\
\text { sie. }\end{array}$ & Coyne et Bellier \\
\hline $\begin{array}{l}\text { Youssef ben } \\
\text { Tachfine. }\end{array}$ & $\begin{array}{l}\text { Direction de l'Hydrauli- } \\
\text { que. Ministère des } \\
\text { vaux Publics et } \\
\text { Communications } \\
\text { Maroc. }\end{array}$ & $\begin{array}{l}\text { SOFRELEC- } \\
\text { Coyne } \\
\text { et Bellier. }\end{array}$ \\
\hline
\end{tabular}

des fuites et par un réseau de piézomètres bien implantés et en nombre suffisant à l'amont comme à l'aval des organes étanches est un élément de la sécurité non moins important. Le dispositif de drainage qui vit avec le barrage et sa fondation au rythme des remplissages et vidanges successifs peut se colmater ou se cimenter, et, par suite, il est nécessaire d'ausculter son évolution pour intervenir à temps par un entretien, un complément ou même un remplacement du système de drainage.

Les études des principaux ouvrages illustrant cet exposé furent le fruit d'une étroite collaboration avec les maîtres d'œuvre. Qu'ils soient remerciés de nous avoir permis de développer les idées nées de la conception de leurs ouvrages.

\section{Bibliographie}

[1] Bertram (G. E.). - Experience with seepage control measures in earth and rockfill dams. $9^{\circ}$ Congrès International des Grands Barrages, Istamboul (1967), vol. III, p. 91.

[2] Bellport (B. P.). - Bureau of Reclamation experience in stabilizing embankment of Fontenelle earth dam. $9^{\circ}$ Congrès International des Grands Barrages, Istamboul (1967), vol. I, p. 67.

[3] KaAmensl (B.) et TorblaA (I.). - Leakage through horizontal cracks in the core of Hyttejuvet dam. Norwegian Geotechnical Institute, publication $\mathrm{n}^{\circ} 80$ (1968).

[4] Vaughan (P. R.) et Leonard (M. W.). - Cracking and erosion of the rolled clay core of Balderhead dam and the remedial works adopted. $10^{\circ}$ Congrès International des Grands Barrages, Montréal (1970), vol. 1, p. 73.

[5] Casagrande(A.), and Wilson (S. D.). - The Baldwin Hills reservoir failure in retrospect. A.S.C.E. Proc. of Specialty Conference on Performance of earth structures, Purdue University (juin 1972), vol. I, part. I, p. 551

[6] Sherard (J.L.) and Decker (R.S.). - Piping in earth dams of dispersive clay. A.S.C.E. Proc. of Specialty Conference on Performance of earth structures, Purdue University (juin 1972), vol. I, part 1, p. 589.

[7] Post (G.) et LuRiN (P.). - Les digues en terre du barrage de Roseires. Annales de l'Institut Technique du Bâtiment et des Travaux Publics (janvier 1970).

[8] Lowe III (J.). - Recent development in the design and construction of earth and rockfill dams. General Report. $10^{\mathrm{e}}$ Congrès International des Grands Barrages, Montréal (1970), vol. V, p. 1.

9] Marchand (R.), Lefebvre (J.) et Gautheron (J.). - Le barrage du Mont-Cenis. Travaux (septembre 1967).

[10] VARGAS (M.) - Effectiveness of cut-offs under 3 earth dams. 4th Panamerican Conference on Soils Mechanics, San Juan (1971), A.S.C.E., vol. II.

[11] Terzaghi (K.). - Design and performance of the Sasumua dam. The Institution of Civil Engineers, paper 6252 (April 1958).

[12] Hamon (M.) et Faliki (F. J.), - Déformation du remblai et fissuration du noyau du barrage de Djatiluhur. $9^{\mathrm{e}}$ Congrès International des Grands Barrages, Istamboul (1967), vol. V, p. 457.

[13] Vaughan (P. R.). - Cracking of clay cores of dams. Surveyor (30 janvier 1970).

[14] Sherard (J. L.) et Decker (R. S.). - Hydraulic fracturing in low dams of dispersive clay. A.S.C.E. Proc. of Specialty Conference on Performance of earth structures, Purdue University (juin 1972), vol. I, part 1, p. 653 .

[15] Casagrande (A.). - Control of seepage through foundations and abutments of dams. Géotechnique (décembre 1961). Discussion par K. TerzAGHr, Géotechnique (mars 1962).

[16] Sabarly (F.). - Les injections et les drainages de fondation de barrages en roches peu perméables. Géotechnique (juin 1968). 
[17] Vaughan (P. R.) et Kennard (N.F.). - Earth pressure at a junction between an embankment dam and a concrete dam. $5^{*}$ Congrès Européen de Mécanique des sols, Madrid (1972), vol. I, p. 215.

[18] Karpoff (K.P.). - The use of laboratory tests to develop design criteria for protective filters. Proc. A.S.T.M., vol. 55, p. $1183-1955$
[19] Kassif (G.) et ZaSLAvSKy (D.). - Analysis of filter requirements for compacted clay. $6^{*}$ Congrès International de la Mécanique des sols, Montréal (1965), vol. II, p. 495.

[20] LEE (K. L.) - Compressibility and crushing of granular soil in anisotropic triaxial compression. Canadian Geotechnical Jounnal (février 1967)

\section{Discussion}

Présidents: MM. BORELI et P. PLICHON

M. le Président remercic $M$. Post pour son exposé qui a montré les multiples solutions imaginées pour s'adapter au mieux à la diversité des conditions de terrain rencontrées. Il ouvre ensuite la discussion.

M. Post commente comme suit le problème de la pérennité dé filtres et des drains évoqué dans l'exposé de M. le Professeur BiAREZ :

C'est là un problème assez sérieux et très mal connu, non parce qu'on n'a pas les instruments d'auscultation (des cellules de pression permettent de voir comment évoluent les pressions dans le drain), mais parce que, la plupart du temps, les barrages n'ont pas donné lieu à fissuration et les fuites évacuées à travers les drains sont extrêmement minimes et loin d'exiger des capacités importantes.

On emploie très souvent, dans les barrages en terre, des matériaux riches en carbonates : le noyau du barrage de Gréoux comportait de l'ordre de 20 à $65 \%$ (dans le matériau criblé à $5 \mathrm{~mm}$ ) de carbonates et celui du barrage homogène de Bir El Cherga de 40 à $55 \%$. Par ailleurs, les voiles d'injection sont aussi une source de carbonates non négligeable. Il suffit de visiter certaines galeries de drainage pour voir à quel point ces carbonates peuvent migrer et venir colmater les filtres; cela présente deux inconvénients avec un double effet : la diminution de la perméabilité et peut-être même un certain effet de cimentation, donc de fragilité qui ferait que ces filtres pourraient se fissurer comme le noyau et ne plus remplir leur rôle.

Je crois qu'il y a là, sur le plan de l'auscultation des ouvrages et aussi sur le plan de la recherche théorique, beaucoup à faire.

M. l'Ingénieur Général DrouHiN (Ministère du Développement Industriel et Scientifique) pose au conférencier la question suivante

Vous avez insisté sur les problèmes de défloculation des argiles et je voudrais savoir si, de votre grande expérience, il résulte que les constructeurs ou les projeteurs font un effort suffisant pour la détermination minéralogique des argiles.

11 est certain que cela n'est pas toujours fait, répond $M$. Post mais il y a des dizaines d'années que nous faisons systématiquement les analyses minéralogiques des argiles utilisées. Je citerai, par exemple, celles du barrage de Rosière, qui comporte un certain pourcentage de montmorillonite. Mais on ne constate aucune corrélation apparente entre le pourcentage de tels ou tels minéraux et les propriétés dispersives de l'argile; notamment, il n'y a aucune corrélation entre les limites d'Atterberg, la limite de liquidité et ces propriétés dispersives.

Je suis persuadé qu'il y a là un champ de recherches extrêmement important et encore assez négligé, conclut $M$. l'Ingénieur Général Droutin entièrement approuvé par M. Post.

M. le Professeur THIRriot intervient en ces termes:

Je voudrais, non pas poser des questions, non pas apporter un complément, mais demander si quelqu'un pourrait faire une synthèse de ce qui a été présenté ce matin. Pour un site donné, il doit y avoir plusieurs solutions. Je pense que certains constructeurs, ou certains représentants des bureaux d'études, ont à l'idée une autre manière d'envisager la solution des problèmes posés.

La confrontation de ces diverses solutions n'aurait-elle pas été un point de départ intéressant pour la discussion; peut-être aurait-elle permis aux non spécialistes de se faire une meilleure idée sur le cheminement qui conduit à la solution retenue.

Il existe pour chaque site de barrage, répond $M$. LonDE, plusieurs «bons projets ». C'est pourquoi l'on constate souvent des différences importantes entre les solutions proposées par différents projeteurs également qualifiés. Mais, il existe aussi de «mauvais projets ». Ce sont ceux qui n'ont pas envisagé toute les hypothèses possibles. Ce sont ceux qui n'ont pas imaginé tous les «scénarios» qu'un site donné permet de bâtir. On a vu, par exemple, dans la conférence de mon collègue Georges Post que les drains et les filtres doivent être conçus en fonction d'éventuelles fissures et pas seulement en fonction de calculs rigoureux de réseaux d'écoulement. Tout le projet, s'il est bon, est marqué par cette méthode : imaginer ce qui peut arriver et s'organiser pour y parer. Il peut y avoir plusieurs réponses satisfaisantes.

M. GiLc (Electro-Watt, Zurich) apporte le point de vue d'un constructeur (Cf. ci-après son mémoire sur la digue de Mattmark)

Après avoir fait une digue dans une situation un peu compliquée, dit-il, on sait ce qu'on refera ct ce qu'on ne refera pas. Il serait extrêmement utile, pour nous tous qui construisons des barrages et des voiles d'injection, de savoir de nos collègues ce qu'ils ne referaient pas et il serait peut-être intéressant de faire un jour un symposium sur ce sujet.

M. L'Ingénieur Général RoвERT intervient ensuite en ces termes

J'ai été très frappé par le mot de «scénario 》 utilisé par M. LONDE et par cet eftort, fait par les constructeurs de barrages, pour imaginer tout ce qui peut, dans la réalité, être différent des hypothèses, nécessairement simplifiées, admises dans l'élaboration d'un projet

D'autre part, $M$. Pos r nous a indiqué que, plusieurs années après la construction d'un barrage qui s'était très bien comporté jusque-là, il peut se passer, parfois assez brutalement, quelque chose de grave. Je voudrais évoquer le problème de l'ouvrage construit, de sa surveillance par les maîtres d'ouvrage, par les autorités qui, lorsqu'il y a un risque public, doivent intervenir. La question que je voudrais poser est celle de savoir quel rôle les ingénieurs-conseils, qui ont scruté minutieusement un projet, étudié différents «scénarios», pourraient jouer pour orienter, conseiller la surveillance et, éventuellement, pour indiquer quels signes précurseurs peuvent alerter sur une situation dangereuse ou susceptible d'évoluer rapidement.

Je me demande même s'il n'y a pas, organiquement, quelque chose à bâtir pour que tout ce qu'ont fait les ingénieurs-conseils puisse être utilisé par les services qui, souvent d'ailleurs, ne sont pas ceux qui ont été les maîtres d'œuvre et qui pourraient coopérer avec les ingénieurs-conseils.

C'est un problème très important et je serais heureux d'avoir à ce sujet le sentiment de M. Post et de M. Londe qui ont une grande expérience.

11 est certain, répond $M$. Post, que le constructeur de barrage, Thomme de l'art comme l'appelle M. Brarez, tire son expérience de l'observation des précédents et, notamment de l'auscultation des différents barrages à la réalisation desquels il a participé, mais aussi de ceux qui ont été construits par ses collègues. Mais, trop souvent, l'ouvrage n'est suivi par le projeteur que pendant la 
première, parfois la deuxième mise en eau, c'est-à-dire pendant les deux premiers cycles de remplissage et de vidange, et son contrat s'arrête là. Je crois que, dans beaucoup de cas, cela n'a aucune conséquence dangereuse, ces ouvrages étant pris en charge par de grands organismes qui ont des moyens d'auscultation et d'interprétation encore plus puissants que ceux des ingénieurs-conseils privés: c'est le cas notamment d'E.D.F. Mais, il y a des cas où le maître d'ouvrage n'a pas les moyens techniques de suivre cette auscultation et il y a là un problème. Il est arrivé - et cela se produit de plus en plus - que des maîtres douvrage passent un contrat avec l'ingénieur-conseil pour suivre périodiquement l'évolution de la vie des barrages conçus par ce dernier. Cette évolution peut se poursuivre fort longtemps, et à ce propos, je voudrais rappeler un exemple étudié récemment par Raoul J. Marsal, mécanicien des sols bien connu; au Mexique, le barrage de Laguna, construit en 1908, s'est rompu en 1968 par érosion régressive à travers sa fondation et ce, sans qu'on puisse expliquer actuellement pourquoi un pareil délai s'est écoulé entre sa mise en service et sa destruction.

Le rôle du projeteur ne se trouve pas terminé à la fin de la construction; mais il ne peut pas suivre tous les ouvrages qu'il a conçus, surtout si on ne le lui demande pas. II n'empêche, qu'avant de remettre un ouvrage à un exploitant, il rédige des règles d'observation de l'ouvrage; il indique des périodes de mesure de différents paramètres: débits de fuite, piézométrie, déformations, etc.; il peut attirer l'attention sur les valeurs-limites au-delà desquelles certains de ces paramètres sont des indicateurs d'un danger. Mais il est toujours très difficile de fixer, a priori, de telles valeurs-limites parce qu'elles dépendent de l'évolution de l'ouvrage au cours des cycles de remplissage-vidange successifs; un paramètre à lui seul n'a pas de sens si l'on ne connaît pas son évolution et sa dérivée.

Certains pays - dont la France - ont édicté des règles très strictes en ce qui concerne le mode d'observation des ouvrages et la périodicité de telles observations.

Intervenant en anglais, le $\mathrm{Dr}$ P. ANAGNOSTr apporte sur ce point d'intéressantes précisions qu'il a résumées comme suit:

Le problème des argiles dispersives est lié à une composition minéralogique particulière de celles-ci et aux propriétés chimiques de l'eau intersticielle. Il existe des méthodes plus ou moins compliquées pour caractériser de tels matériaux avant leur utilisation dans les digues. Cependant, il existe des sites où on ne dispose d'aucun autre matériau; on doit alors utiliser même des argiles potentiellement dispersives mais en prenant des mesures de sécurité supplémentaires dans le profil de la digue. Dans la plupart des cas, l'épaisse zone du filtre amont, constituée par un sable à grain fin sans cohésion, procurera une bonne protection contre la formation de cavités ou de grande fissures ouvertes dans la zone des argiles dispersives. La protection est réalisée par un transport hydraulique des particules fines du filtre vers les fissures, ce qui assure le comblement de celles-ci et la réduction des vitesses d'écoulement. Une protection analogue peut être assurée sur les talus extérieurs de la digue en disposant une couche granulaire, riche en matériaux fins sans cohésion qui préviendront l'approfondissement des érosions provoquées par le ruissellement des pluies.

\section{Posr répond en ces termes:}

Je n'ai pas dit que j'étais particulièrement inquiété par le problème des argiles dispersives, mais simplement que ce problème était très mal connu et qu'il fallait l'examiner de facon attentive. L'essai de dispersion très simple imaginé par R. A. Rallings (Australie), en 1966, - que nous employons de façon régulière — ne suffit pas à établir une corrélation complète qui permettrait de séparer les argiles dangereuses et celles qui ne le sont pas. Les phénomènes d'érosion par l'eftet des pluies, amorcés à l'occasion de la fissuration de surface, sont bien connus et, souvent, sont la conséquence des propriétés dispersives des terres argileuses. I1 est certain qu'une couche non cohérente de sable, en évitant la dessiccation et la fissuration initiale de ces terres, remédie de façon convenable à ce risque. Il existe d'ailleurs d'autres movens de le pallier : on peut traiter la couche supérieure du massif par de la chaux; avec 4 à $5 \%$ de chaux on arrive à avoir une couche non dis- persive qui ne se fissure pas et qui, par conséquent, évite l'évaporation des matériaux sous-jacents et leur fissuration initiale.

Un autre moyen, qui a été appliqué sur certains petits barrages, consiste, non plus à traiter le sol par de la chaux, mais à traiter l'eau infiltrée; il consiste à interposer entre le filtre et le noyau une couche de gypse qui enrichit l'eau en ions calcium dans cette zone, rendant ainsi très faible le risque de dispersion des argiles.

Il existe donc un arsenal de moyens pour lutter contre ces risques, encore qu'ils ne soient pas bon marché. La couche supplémentaire en gypse et l'addition de chaux dans le noyau sont, en fait, des moyens de traitement des cas extrêmes.

Ce qui est très important, c'est d'avoir à l'amont un filtre abso lument pulvérulent sans aucune cohésion de façon que la fissuration ne puisse pas se transmettre.

Concernant le risque de défloculation, je voudrais citer un cas limite qui est celui du barrage norvégien de Hyttejuvet; cet ouvrage d'environ $100 \mathrm{~m}$ de hauteur, comporte un noyau très mince qui a été aminci d'ailleurs en cours de construction en raison des pressions intersticiełles excessives qu'on y a observé. On craignait pour la stabilité à partir d'un certain niveau et on a aminci lo noyau, lequel présente un gradient de l'ordre de 4,5 dans la partie supérieure. Ce noyau est constitué par un matériau morainique de granulométrie extrêmement continue mais qui renferme peut-être un excès de fines ( 30 à $35 \%$, inférieur à $80 \mu$ ) et pratiquement pas plastiques (indices de plasticité de l'ordre de 5 à $6 \%$ ). Il y a ell un effet de transfert de charge entre ce noyau et les recharges qui sont séparées par un filtre tout à fait classique de chaque côté l'ensemble répond tout à fait aux normes, les recharges étant constituées par des alluvions de transition et des enrochements. Malgré cela, il y a eu fissuration du noyau à la mise en eau par claquage hydraulique dans une région décomprimée par «effet d'arc» et les fines défloculées sont passées à travers le filtre.

On se trouve là vraiment dans un cas extrême fort différent de celui des matériaux argileux classiques. Est-ce à dire que c'est un matériau dispersif au sens défini par J. S. Sherard, c'est-à-dire ayant un fort pourcentage de dispersion? Il semble bien : les essais avec de l'eau distillée ont donné un pourcentage de particules inférieures à $5 \mu$ de l'ordre de $50 \%$ de celui obtenu par essai standard après addition d'un défloculant. Donc le problème n'est pas si simple et, s'il ne semble pas impliquer un risque sérieux, il constitue un champ d'étude important.

Pour terminer, M. le Professeur Thirriot pose une question qu'il qualifie «d'apéritive» en ce sens qu'elle peut être discutée au cours du repas dont l'heure s'approche.

Depuis un jour et demi, dit-il, nous recevons des informations sur des problèmes, des méthodes et des réalisations en profitant de l'expérience des uns et des autres. Le moment semble venu de poursuivre cet échange dans une optique prospective. En particulier, puisque cette session est placée sous le patronage commun de la Société Hydrotechnique de France et de l'Association Internationale de Recherches Hydrauliques, je voudrais demander à tous les participants de réfléchir au problème de la recherche hydraulique et, en particulier, d'apporter une collaboration bénévole aux laboratoires universitaires - et peut-être même à d'autres laboratoires de recherche - qui en sont réduits à étudier encore les écoulements à travers les digues homogènes à parements verticaux. Il y a certainement d'autres problèmes à élucider. Compte tenu du fait qu'au moins sept ou huit laboratoires européens sont représentés ici (dont plusieurs laboratoires universitaires), je suis sûr que les suggestions qui leur seront faites par les constructeurs, en vue d'examiner les questions qu'eux mêmes n'auraient pas le temps d'étudier ou dont le thème ne rentre pas exactement dans leur vocation, seraient certainement prises en considération.

En fait, il s'agit de cerner quelques thèmes suffisamment précis qui pourraient être donnés en pâture aux laboratoires afin de rapprocher leurs préoccupations, dans ce domaine, de celles des constructeurs.

M. Le Président remercie les rapporteurs, l'auditoire qui a suiv tous les exposés avec beaucoup d'attention et toutes les personnes qui ont participé à la discussion.

La séance est levée à $12 \mathrm{~h} 20$. 